\title{
Cardiogenic Shock as an Initial Presentation of Addison's disease: A Case Report
}

Sara Belabyad*, Mohammed Anas Khouchab, Zainab Boudhar, Dounia benzeroual, Saloua El Karimi, Mustapha El Hattaoui

Cardiology Department, Mohammed VI University Hospital, Marrakesh

DOI: $10.36347 /$ sasjm.2020.v06i01.003

| Received: 05.01.2020 | Accepted: 12.01.2020 | Published: 22.01.2020

*Corresponding author: Sara Belabyad

Abstract

Addison's disease, also known as primary adrenal insufficiency, is an endocrinological condition with a deficiency in the production of glucocorticoids and mineralocorticoids from the adrenal cortex. It's a very life-threatening disease that is often overlooked due to the unspecific range of symptoms patients usually present with, thus a prompt diagnosis is essential. We present the clinical history, physical findings, laboratory results, and imaging studies of a young female presenting with cardiogenic shock revealing Addisson's disease. The association between adrenal insufficiency and cardiogenic shock and pertinent literature are reviewed.

Keywords: Addison's disease, adrenal insufficiency, glucocorticoid, cardiogenic shock.

Copyright @ 2020: This is an open-access article distributed under the terms of the Creative Commons Attribution license which permits unrestricted use, distribution, and reproduction in any medium for non-commercial use (NonCommercial, or CC-BY-NC) provided the original author and source are credited.

\section{INTRODUCTION}

\begin{abstract}
Addison's disease is associated with a decreased production of glucocorticoid and mineralocorticoid hormones from the adrenal cortex [1, 2]. Although autoimmune adrenalitis is considered to be the major cause of Addison's disease in up to $90 \%$ of diagnosed individuals, prevalent in female patients between 30 and 50 years of age, other etiologies include infectious, drug induced, and/or genetic factors [3, 4].Common manifestations of this condition are hyponatremia, hyperkalemia, and/or hypoglycemia along with mucosal and skin hyperpigmentations [3-5]. Adrenal insufficiency usually presents with vague symptoms such as weakness, fatigue, anorexia, weight loss, and hyperpigmentation as the primary symptoms. Although cardiovascular manifestations of Addison's disease include hypotension, syncope, and arrhythmias, the development of a dilated cardiomyopathy and heart failure are an uncommon life-threatening complication [6-10]. Cardiogenic shock arising from adrenal insufficiency is less common $[4,5]$.
\end{abstract}

We describe a unique case of a young female with undiagnosed primary adrenal insufficiency presenting with cardiogenic shock.

\section{CASE REPORT}

We received a 47 year-old female patient with a history of severe congestive heart failure and sever systolic dysfunction, with no history of hypertension, diabetes mellitus or smoking. She was under furosemide $40 \mathrm{mg}$ twice daily, ramipril $10 \mathrm{mg}$ daily, bisoprolol $5 \mathrm{mg}$ daily, spironolactone $50 \mathrm{mg}$ daily and omeprazol $20 \mathrm{mg}$ daily. She had not been taking any oral contraceptives, or any natural medicines. The patient was admitted to our hospital due to the on-set of dyspnea (NYHA class III) with anasarca two weeks prior to her admission. She reported orthopnea, paroxysmal nocturnal dyspnea and decreased appetite. On presentation, the patient's vital signs were: blood pressure $75 / 35 \mathrm{mmHg}$, heart rate $153 \mathrm{bpm}$, respiration rate 30 breaths/min, saturating $85 \%$ on room air and body temperature was $37^{\circ} \mathrm{C}$. On physical exam, the patient had the clinical signs of florid heart failure with edema of the lower extremities, cool extremities, abdominal distention, tachycardia and neck vein distension, with exhibited generalized hyperpigmentation and tanning of her skin creases. On chest auscultation, there were bilateral rales audible over the lower two thirds of both lung fields. The patient was in cardiogenic shock with systolic pressures in the $75-80 \mathrm{mmHg}$ range and oliguria. Transthoracic echocardiography (TTE) confirmed a dilated left ventricle (LV), severe LV systolic dysfunction with an 
ejection fraction of $25 \%$ and low cardiac index (1.2 $1 / \mathrm{min} / \mathrm{m} 2$ ), with moderate functional mitral regurgitation and global hypokinesia. The inferior vena cava was dilated to $29 \mathrm{~mm}$ with sever pulmonary hypertension at $80 \mathrm{mmhg}$. Chest $\mathrm{X}$-ray findings were consistent with bilateral pleural effusions, vascular redistribution, and interstitial edema with cardiomegaly (Fig 1). Her electrocardiogram (ECG) showed atrial fibrillation (Fig 2). Laboratory data showed a low sodium level of $132 \mathrm{mmol} / \mathrm{L}$ (normal range: 135-145 $\mathrm{mmol} / \mathrm{L}$ ), a normal potassium level of $4.7 \mathrm{mmol} / \mathrm{L}$ (normal range: $3.5-5.0 \mathrm{mmol} / \mathrm{L}$ ). Blood urea and creatinine levels were also normal, with normal blood glucose and white cell counts. Cardiac troponins were high, at $30 \mathrm{ng} / \mathrm{dl}$ with negative kinetics. Creatine kinase level was elevated. Blood and urine cultures were negative

Believing inotropic support with dobutamine would improve cardiac output and lead to significant unloading and subsequent diuresis, a continuous dobutamine infusion at $10 \mathrm{mg} / \mathrm{kg} / \mathrm{min}$ and a continuous furesemide infusion at $240 \mathrm{mg} / 24 \mathrm{~h}$ were begun.

To further evaluate her vasopressor resistant hypotension, an early morning cortisol was checked and was low at 23.7ug/L (normal range: 62-194ug/L). Subsequently, adrenocorticotrophic hormone levels were elevated; which is in favor of Addison's disease. Her free thyroxine and her thyroid-stimulating hormone were normal. She was treated with hydrocortisone 100 mg every 6 hours. Her clinical status and hemodynamics greatly improved. Steroids were weaned to physiologic doses of hydrocortisone $10 \mathrm{mg}$ in the morning and $5 \mathrm{mg}$ in the evening.

Nine days after admission to the ICU, she was transferred to a normal ward. Throughout the further hospital stay, her clinical condition improved rapidly. She was discharged with standard replacement doses of hydrocortisone.

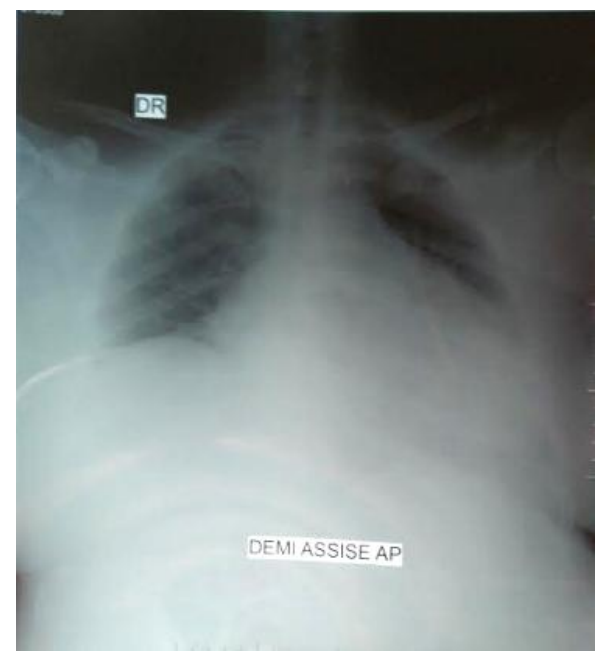

Fig-1: Chest X-ray revealed prominent cardiomegaly with increased pulmonary vascularity on both lung fields

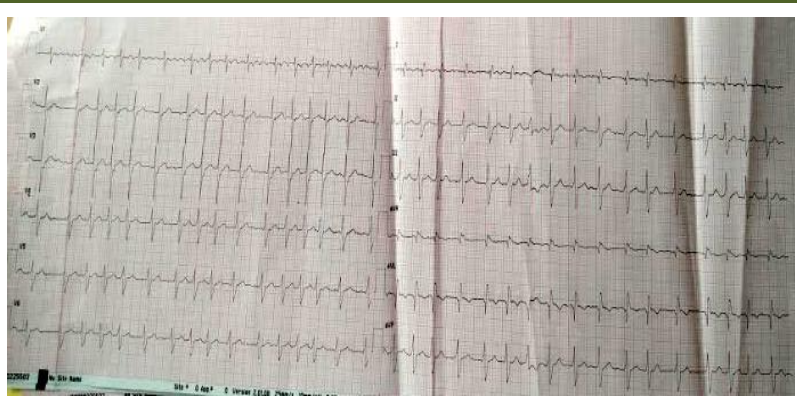

Fig-2: ECG at admission showing atrial fibrillation

\section{DISCUSSION}

With an incidence of approximately 120 cases per million in the population, Addison's disease is a rare long term endocrine disorder in which the adrenal glands produce insufficient steroid hormones, including glucocorticoids (GCs) and mineralocorticoids [1-4, 10]. Addison disease has many causes, the most common in the Western world being autoimmune adrenalitis, which results from destruction of the adrenal cortex.

Cortisol can act either directly or indirectly on the peripheral vessels and heart tissue by potentiation of vasoactive factors [5, 11]. Aldosterone has also been implicated as a direct regulator of cardiac extracellular matrix and collagen deposition [5, 12]. Although an excess of these hormones is associated with cardiac abnormalities in the form of myocardial hypertrophy and dysfunction, little is known about the effects on the myocardium when production of adrenal steroids is impaired $[13,14]$.

The direct effects of GCs on the myocardium are difficult to study, as changes in plasma GC concentrations have consequences due to the pervasive expression of the GC receptor, resulting in secondary effects on myocytes [15]. The existing literature about the effects of low corticosteroid hormones (aldosterone, GC hormones) on cardiac function is limited.

The etiology of adrenal insufficiency is believed to be a result of increased serum levels of cytokines interleukin-1a, interleukin-6, and tumor necrosis factor-a, associated with severe illness. These cytokines blunt the response of the hypothalamuspituitary- adrenal axis and increase local tissue resistance to cortisol [4, 15]. Most cell types express glucocorticoid receptors in the cytoplasm enabling glucocorticoids to cross the cell membrane, bind intracellular receptors, translocate to the nucleus, and alter gene transcription. Glucocorticoids potentiate the effects of catecholamines contributing to vascular tone and cardiac contractility. They are also antiinflammatory, as well as immunosuppressive agents [15]. The pathophysiology of adrenal insufficiency has yet to be clearly defined, but there appears to be a shortage of corticosteroids resulting in hemodynamic instability. 
Cardiac failure has been described as a coexisting illness in adrenal insufficiency in an earlier case report [5, 16]. Profound hypotension has been shown to be refractory to catecholamines because it is also associated with secondary volume depletion due to adrenal failure [16].The myocardial dysfunction and severe hypotension may lead to a fatal course if underlying adrenal insufficiency is unrecognized and left untreated, whereas GC treatment rapidly improves the myocardial dysfunction within several days to weeks [5-17].

It has been reported that adrenalectomized rats have impaired myocardial contractility, which was found to be associated with a depletion of microsomal phosphorylase activity and reduction in calcium uptake in the sarcoplasmic reticulum [18, 19]. GC deficiency may downregulate expression of adrenergic receptors, decrease the synthesis of adrenaline, and then also decrease the cardiovascular reactivity to catecholamines. It is hypothesized that GC deficiency may result in the loss of protective effects against catecholamines. These underlying mechanisms could explain the severe myocardial dysfunction associated with adrenal insufficiency $[20,21]$.

Cardiac failure leading to death is well recognized as a major problem in adrenal crisis. There are several hypothesized mechanisms to explain the cause of the cardiac failure [15]. Hypovolemia resulting from mineralocorticoid deficiency alone seems insufficient to explain the sudden profound cardiovascular collapse in our patient. The electrolyte abnormalities causing compromised myocardial performance may be important, but experimental evidence has shown that they are not decisive in severe adrenal insufficiency. Sympathectomy and alpha/betablockade have been shown to protect stressed adrenalectomized animals from circulatory failure [5$18]$.

This report describes relative adrenal insufficiency in decompensated heart failure complicating cardiogenic shock. Relative adrenal insufficiency has been described in certain subsets of critically ill patients, but never in end-stage heart failure as a cause of critical illness and shock. Hemodynamic compromise in endstage heart failure and cardiogenic shock has been studied in a number of trials [22].

This report details a case of cardiogenic shock that responded to corticosteroid replacement identifying what we believe is another group of patients that will benefit from diagnosis of the syndrome of relative adrenal insufficiency and appropriate steroid replacement [22].

The diagnosis of adrenal insufficiency in this patient population may ultimately improve outcomes, especially in patients with end-stage heart failure complicated by cardiogenic shock. It is likely that this syndrome is undiagnosed and it is our hope that a clinical trial will be performed to ultimately determine the prevalence of this syndrome in end-stage heart failure and cardiogenic shock, as well as define the 'appropriate' levels of cortisol at baseline and the 'appropriate' response to steroid replacement

\section{CONCLUSION}

Due to nonspecific symptoms on presentation, primary adrenal insufficiency can pose a challenge to diagnose. Although the presentation of cardiogenic shock in a patient with undiagnosed adrenal insufficiency is considered a rarity, with hypovolemic shock being more common, practitioners should consider adrenal insufficiency in the differential diagnosis for cardiogenic shock without a delay in instituting stress-dose steroids.

\section{REFERENCES}

1. Viktoriya Mozolevska, Anna Schwartz and al. Addison's Disease and Dilated Cardiomyopathy: A Case Report and Review of the Literature, Case Reports in Cardiology Volume 2016, Article ID 4362514, 5 pages http://dx.doi.org/10.1155/2016/4362514

2. Nieman LK, Turner ML. Addison's disease. Clinics in Dermatology. 2006 Jul 1;24(4):276-80.

3. Bensing S, Hulting AL, Husebye ES, Kämpe O, Løvås K. Management of endocrine disease: epidemiology, quality of life and complications of primary adrenal insufficiency: a review. European Journal of Endocrinology. 2016 Sep 1;175(3):R107-16.

4. Charmandari E, Nicolaides NC, Chrousos GP. Adrenal insufficiency. The Lancet. 2014 Jun 21;383(9935):2152-67.

5. Lohiya S, Subramanyam S, Ovalle F. Cardiogenic Shock: An Initial Presentation of Primary Adrenal Insufficiency. AACE Clinical Case Reports. 2016 Jun;2(3):e206-9.

6. CUSHNER GB, ZAHLER SF, HILLS AG. Untreated Addison's disease complicated by pulmonary congestion due to left ventricular failure. Annals of internal medicine. $1963 \mathrm{Feb}$ 1;58(2):341-6.

7. Bhattacharyya A, Tymms DJ. Heart failure with fludrocortisone in Addison's disease. Journal of the Royal Society of Medicine. 1998 Aug;91(8):433.

8. Afzal A, Khaja F. Reversible cardiomyopathy associated with Addison's disease. The Canadian journal of cardiology. 2000 Mar;16(3):377-9.

9. Wolff B, Machill K, Schulzki I, Schumacher D, Werner D. Acute reversible cardiomyopathy with cardiogenic shock in a patient with Addisonian crisis: a case report. International journal of cardiology. 2007 Mar 20;116(2):e71-3.

10. Krishnamoorthy A, Mentz RJ, Hyland KA, McMillan EB, Patel CB, Milano CA, Hernandez AF. A crisis of the heart: an acute reversible 
cardiomyopathy bridged to recovery in a patient with Addison's disease. Asaio Journal. 2013 Nov 1;59(6):668-70.

11. Fraser R, Davies DL, Connell JM. Hormones and hypertension. Clin Endocrinol (Oxf). 1989;31:701746.

12. Young M, Fullerton M, Dilley R, Funder J. Mineralocorticoids, hypertension, and cardiac fibrosis. J Clin Invest. 1994;93:2578-2583.

13. Fallo F, Budano S, Sonino $\mathrm{N}$ and al. Left ventricular structural characteristics in Cushing's syndrome. J Hum Hypertens. 1994;8:509-513

14. Rossi GP, Sacchetto A, Pavan E, Palatini P, Graniero GR, Canali C, Pessina AC. Remodeling of the left ventricle in primary aldosteronism due to Conn's adenoma. Circulation. 1997 Mar 18;95(6):1471-8.

15. Sainte-Marie Y, Cat AN, Perrier R, Mangin L, Soukaseum C, Peuchmaur M, Tronche F, Farman N, Escoubet B, Benitah JP, Jaisser F. Conditional glucocorticoid receptor expression in the heart induces atrio-ventricular block. The FASEB Journal. 2007 Oct;21(12):3133-41.

16. Walker C, Butt W. Case of cardiovascular collapse due to adrenal insufficiency. Aust Paediatr J. 1988;24:197-198

17. Shimizu M, Monguchi T, Takano $\mathrm{T}$ and al. Isolated ACTH deficiency presenting with severe myocardial dysfunction. J Cardiol Cases. 2011;4:e26-e30.

18. Narayanan N. Effects of adrenalectomy and in vivo administration of dexamethasone on ATPdependent calcium accumulation by sarcoplasmic reticulum from rat heart. J Mol Cell Cardiol. 1983; $15: 7-15$

19. Rao MK, Xu A, Narayanan N. Glucocorticoid modulation of protein phosphorylation and sarcoplasmic reticulum function in rat myocardium. Am J Physiol Heart Circ Physiol. 2001;281:H325-H333.

20. Collins S, Caron MG, Lefkowitz RJ. $\beta$-Adrenergic receptors in hamster smooth muscle cells are transcriptionally regulated by glucocorticoids; J Biol Chem. 1988;263:9067-9070.

21. Orlowski J, Lingrel JB. Thyroid and glucocorticoid hormones regulate the expression of multiple $\mathrm{Na}$, $\mathrm{K}$-ATPase genes in cultured neonatal rat cardiac myocytes. J Biol Chem. 1990;265:3462-3470

22. Lovelock JD, Coslet S, Johnson M, Rich S, Gomberg-Maitland M. Relative adrenal insufficiency in severe congestive heart failure with preserved systolic function: a case report. Journal of Cardiovascular Medicine. 2007 Sep 1;8(9):7547. 